American Journal of Law and Legal Studies
(ISSN:2638-2202)

\title{
WEB INFORMATION QUALITY VERSUS TURBULENCE ON URL DECAY: CRITIC ON RELIABILITY OF AUTHORITY CITATIONS
}

\author{
Zharama M. Llarena \\ Juris Doctor student, California Southern University
}

\begin{abstract}
Internet usage provides environment for learning, research, ${ }^{*}$ Correspondence to Author: entertainment, and business. Web design is crucial for market- Zharama M. Llarena ability of the Web page, whereas, Web information is essential Juris Doctor student, California for enhancing research skills for academic and institutional Southern University growth and development. This study aims to assess information provided in the various Internet sites according to several literature content compliance and gaps on URL forming How to cite this article:

rots over time as inaccessible URLs may be subject to copyright Zharama M. Llarena. WEB INinfringement. References of authors render article citations by FORMATION QUALITY VERSUS providing URLs, DOIs, and PURLs, in which old URLs tend to TURBULENCE ON URL DECAY: be unavailable as time function which may be subject to CRITIC ON RELIABILITY OF AUintellectual property issue by communication obstruction of THORITY CITATIONS.American public right. Hyperlinks enable end-users to access Web Journal of Law and Legal Studies, content for an exclusive communication right to the public 2020, 3:9

under Article 8 of WIPO Copyright Treaty as retrieval from Internet Archives and Google services had a limited ability to preserve its full content, hence, public communication results to impediment. Therefore, modern DOls and PURLs must be preferred in citing articles as their unique identifiers are permanent, unlike old URLs that used to rot after several years.

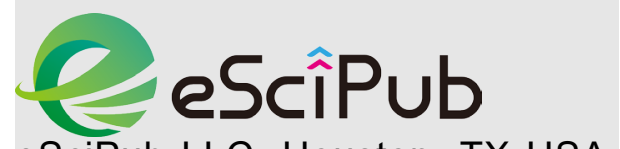
Keywords: website, citation, references, archiving, intellectual property
\end{abstract}




\section{INTRODUCTION}

One of the most crucial and intricate historical inventions for the benefit of mankind is the Internet ${ }^{1}$. Design quality is commonly under the control and management of a Web developer, whereas, data quality is evaluated by various experts from several study domains ${ }^{2}$. A lot of users gain access with the Internet due to intent of acquiring ample benefits from leisure and news update to learning and research. In line with Internet development, the full texts of many scientific journals and articles are provided electronically and via the form of open access for researchers, hence, the Internet has been recognized as one of the major communicational tools for technical users. Hence, e-databases, e-journals, e-books, e-theses, e-prints of research documents, etc., have rendered purview for technical users in many areas of cognitive domains and motivated their research proficiency. As an outcome, usage of references based from old URLs for citations as resources from Internet had augmented. The major determinant for increment of scholarly papers in Web citations in Learner Information System (LIS) is that the Web has been the first opt of technical users for searching research data, creating scientific studies and communicating with foreign colleagues at other research institutions. Meanwhile, there are millions of open access resources accessible on the Web that resulted LIS authors to suggest it to another more Web resources in order to play their role of enhancing research proficiency ${ }^{1}$.

The Internet use in academic environment has provided easy availability to several resources, and information dissemination has, hence, significantly augmented. Furthermore, the prevalence of this distribution has led to supplemental advantages in that these resources can be utilized in any time and place. Despite of technological efficiency, it is assessed with proportional usage of intended outcomes in learner's achievement does not precisely liberate and is complexed to ascertain. Therefore, much research has been facilitated through time to determine the factors for this problem ${ }^{3}$.

Some researches recommended that learners must commonly tend to utilize search engines rather than e-libraries to review the literature concerning their assignment or project expertise. Brophy \& Bawden (2005) made a comparison between Google as an Internet tool for search engine with academic library resources in their investigation. Their similar result of precision surprisingly exhibited that while Google is higher in scope and availability, library systems are higher for quality of outcomes. Lastly, they generalized that the good use of joined purview is crucial since both have many novel tools. Lazonder (2000) studied the novice researchers' training requirements in looking for information on the World Wide Web, citing the page location is more essential than searching the information on a search engine ${ }^{3}$.

The Internet and computers are the most efficient communication resources, which constitute a portion of our daily lives and serve as major tools in education. The Internet assists in transferring of information between varying points, hence, this completion creates the Web as a strong persuasive tool in data system. People in varying age groups, learners and mentors who make scientific research and study projects tend to use the Internet since it is the simplest, quickest, and lowest price means of acquiring important information access ${ }^{3}$.

Although the Internet is a very crucial and necessary source for students, there is a raised problem whether the cited source is credible or trustworthy or both. This is due to the reason that there is no regulation on any specific information piece published via the Web, in contrast to the professional and scientific journals published by the scientific organizations, business field and public institutions. Furthermore, other journals and books released by commercial institutions do not have a regulatory unit constituting editors and referees. Several Internet sites provide access to any person for submission of any information type lacking any personal restriction, and 
numerous reliable sites are limited to open access for commercial intents or security needs ${ }^{3}$.

Information accuracy pertains to one of the most crucial features of academic writing, as citing related published journals is one case example. Certainly, references are crucial in the scientific knowledge flow. Appropriate reference citation allows end-users to look for further information on focused topic ${ }^{4}$.

There have been many issues pertaining to the citation accuracy being published. Reference accuracy comprises exact citation to all fields of identification, such as the journal title, publication volume or year, and the start page article number, the mistake in reporting specific elements that will definitely lead in retrieval difficulties, even impossibilities, to track the original source. Former studies on citation accuracy in a wide scope of general and specialty biomedical journals published error rates ranging from 10\% to $70 \%{ }^{4}$.

The general aim of this study is to evaluate websites of several domain suffixes in accordance with invariance and issues relating to concepts on tortfeasance and compare it to various degree of literatures and contrast its application to information sources.

\section{PREMISES}

Citations of articles render many reasons of intent. Authors utilize citations to commend other writers' concept. Analysis of citations is utilized to observe trends in a specific domain. Technical users make citations a way of looking original or supplemental information sources ${ }^{4}$.

The Internet utilization, together with the World Wide Web development, for determining essential information, has been a crucial means for several scientists all over the world, as numerous scientific domains, such as molecular biology and immunology, are quickly modifying as several works are daily provided on the Internet in a digital format. Hence, the biomedical community has acquired the Internet as a readily accessible forum to disseminate several file formats, inappropriate for print media like videos, software programs, huge databases, sound, etc. With augmenting access on scientific Internet articles, one referencing types, made popular as time function, is website citations (URLs) in order to currently commend $1 \%$ to $19 \%$ articles of at least one URL. Nevertheless, the information from Web pages may be inaccessible over time, whereas, printed articles and books are retrieved in libraries, since old URLs cited within scientific and medical literatures tend to rot through time. There are also problems regarding the credibility and quality of articles provided in the Web. Mainstream and small medical journals vary in many features. Majority of writers in small medical journals originate from developing countries wherein access to subscription-based databases, may not be readily available. Most researches facilitated on referencing patterns to URLs have analyzed published journals from developing countries ${ }^{4}$.

Link rot, also termed as Uniform Resource Locator (URL) decay, has been an increasing problem in biomedical sciences domain. Generally, URL decay happens for numerous reasons, such as servers may close due to business failures, URL content may be reconfigured or modified by the owners of Web sites, and mistakes in URL citations may take place. Numerous articles have attempted to report the URL decay extent in scientific literature. A 2002 article evaluating Web site access after three years of its shown initial availability, "over $66 \%$ could not be retrieved anymore or had transferred lacking forwarding URL." Wren in 2004 commended that only $78 \%$ of published MEDLINE abstracts in URLs were commonly accessed at the accession time. Latest articles put URL access at approximately $70 \%$ with a yearly decay rate of $5.4 \%$. A generalization in all studies is a high link rot rate for biomedical journals. Due to published access in URL over time, it is anticipated that unavailability of URL links will worsen unless provided measures are shown to mitigate the problem ${ }^{5}$. 
Numerous treatments were offered leading to $U R L$ decay reduction. One is the Digital Object Identifiers (DOI) usage which are novel and permanent identifiers for URL accompaniment. DOIs are provided to an electronic document which, opposite to a URL, is independent upon the location of an e-document. It is utilized to render updated data, comprising the object location in the Web, services concerning the object, or any other described information piece. URL data may alter over time, but not $\mathrm{DOI}$ and hence, its location is permanent. DOIs can be utilized to ascertain e-texts, images, video or audio items, data-bases and software. Crossref, a separate membership association discovered and managed by publishers, facilitates a cross-publisher citation linking system that permits technical user to open commended reference on one platform of the publisher and associate directly to the cited data on another platform of the publisher. Currently, this citation-linking network documented millions of journals coverage and other information items from numerous scholarly and professional publishers ${ }^{5}$.

The Online Computer Library Center proposed another means known as the Persistent Uniform Resource Locator or PURL usage. PURL, like $\mathrm{DOI}$, is a unique and permanent address. Any alterations in the material content or organization of publishers will only require an update in the server of PURL ${ }^{5}$.

Google services and Internet Archive are accessible to researchers experiencing inactive Internet references. Internet Archive is a non-profit organization that renders free availability to a digital library of Web sites. Google renders hoarded Web pages. However, these services do not provide ideal retrieval since they may only archive $33 \%$ to $50 \%$ of the total Web pages. Furthermore, the retrieval page may not be the actual Internet page made available and commended originally by the writer, therefore, may not comprise the actual content. Finally, retrieval pages do not comprise large applications or databases ${ }^{5}$.
Approaches of utilizing institutional resources are essential for institutional research. The comparison between journals on the focus of interest can be utilized to organize them for literature review. Clustering of journals in detail assists to maintain the anticipated text originality resulting to project achievement ${ }^{3}$.

\section{Academic Journals Database}

The Academic Journals Database efficiency is associated to several periodicals covering and assessing their methods resulted to creation of a universal academic index. They render an ample specialized information sources which are utilized by many learners and mentors. They are crucial reference tools for these researchers in order to conduct detailed study into various subject areas and are clustered into topics of interest in accordance to varying disciplines ${ }^{3}$.

It is anticipated that there must be same equivalency between prestigious and regional articles regarding citation pattern to URLs and their rot through time, as unavailability of the Internet citations is an intrinsic assumptive problem with the Web site and not be associated to the journal type, impact factor, or reader base. Despite of the previous study, mainstream articles vary from low-profile articles concerning both reference pattern of Web site citations and rot of URLs that could be presumably be described by the fact that the writers publishing in these two journal types are apparently different. The writers of the regional journals are chiefly from developing countries and may not be readily available to subscription-based databases, such as Scopus, Web of Science, EMBASE, etc. The quality and credibility of the Web sites these writers have acquired availability and pertained to may not be equivalent to the high regard those generally published citations by authors of prestigious medical schools. These lower in equivalency writers are commonly studying in large research centers in developing countries with ready availability to several databases, software programs, and IT consultants ${ }^{3}$. 


\section{Search Engines}

Search engines are open access sites having the most broadly used resources for project learners. Many open access articles are available on Internet Explorer programs, while some tools are fitered by rendering multiple search engines such as "Copernic". Resources which are made available via these engines are majorly used by learners for collecting the needed project data. Information acquired from these sites alters according to learner's level and the project information importance. By various customization in the advanced search tabs, direct available resources render more efficient query results for particular document types like files with pdf, doc, ppt extensions. These resources resulted to a feasible availability of data content needed to remove undesired resources. Although compelling trap/pop-up sites tend to catch the learner's notice, they convert them into their consumed time on varying topics of interest in varied sites while studying on a project ${ }^{3}$.

\section{Electronic Libraries}

Electronic libraries which provide a crucial benefit in making the content to be readily available needed from associated sites are grouped into two different types: open or restricted access university Web sites, and other pages which are entirely open via the World Wide Web. The restricted sources in e-libraries in academic institutions are grounded on contractual journals and academic books with absolute trustworthiness. These cluster of references are ideal for people affiliated to a university like researchers, learners, and mentors. Although the open access reliability of libraries is an inappropriate level, it is estimated with the same reliability level as external sources that authored them. The Wikipedia is an official research tool made to compare contents with other resources, making it a more detailed, reliable articles in libraries. Due to this reason, most topics of interest in Wikipedia are authored by official and private libraries, even though the article reliability can only be evaluated, if the citations have been well referenced ${ }^{3}$.

\section{Blogs/Forums}

Several students are able to acquire availability of the needed contents by utilizing search engines for project assignments. Many topics of interest included in search engines are forums and written blogs. The content provided in these links is commonly be found to be acceptable by the readers. As an outcome, a comparison must be made after application of various sources and contents must have confirmation. These openaccess resources are usually personal opinions of people who are focused on a subject, disregarding whether the writers have academic proficiency or none, and hence, must have confirmation with other sources on the same topic of interest by people who are legally eligible to explain those concepts ${ }^{3}$.

\section{Distance Learning}

Via the current usage of advanced technologies, students can learn anytime, anywhere by ways of remote education, where the student-teacher relationship is performed at simultaneous or varied times lacking the need for physical presence. The remote education infrastructure is grounded on Internet-based training, so that the current content for dependability and importance relies on how trustworthy and wellfacilitated the content corporation management is. Data management systems must be versatile, simple, trusted by public, and with a supportive cultural learning ${ }^{3}$.

\section{Related Software}

There are several and different software programs utilized by students of varying university departments. They are utilized as a current part of academic program or associated to a particular scientific branch subsequent to graduation. This software comprises the needed content for both industrial life and projects. Several computer programs are for commercial intents but should be utilized under license and their use is very restricted for academic intents ${ }^{3}$. 


\section{DISCUSSION}

The prediction that a URL would be inaccessible was significantly linked with augmenting time from publication, and greater depth of directory, but not with the tilde presence or date of accession. These links corroborate Casserly and Byrd's results in their journals of data science. From decayed URLs, $58.8 \%$ were archived in some form in the IA, and an evaluated information of randomly chosen URLs exhibited a relevant content of information. This research also supports that $12 \%$ of URLs in MEDLINE abstracts comprise technical mistakes that provide the published URL inaccessible ${ }^{6}$.

Another issue is the copyright infringement possibility linked with Internet content preservation which is not the intellectual property of the commended author. In an instance concerning scientific publications, a recent paper exhibited that several authors make journal reprints accessible online, which may in turn be retrieved by the IA without regard of the condition concerning the wants of the articles and its content to be freely accessible. It is complex, if feasible, in several cases for the for the IA to determine what information has been legally and illegally posted. Web authors may inquire to have their electronic information extracted from the IA, which may further restrict the IA ability to preserve URLs ${ }^{6}$.

Hyperlinks associate Web users to information locating on the Internet. "Simple" links transfer the user to a home page from which the individual may navigate to particular works, such as "deep" and "in-line" or "framing" links transfer the user directly to the information the user is looking, in the latter situation by providing the information "framed" by the website the user first communicated to track the works by request. Linking, which was once a crucial way of Internet navigation, but also a frequent way to access copyright infringement, challenges courts to explain the legal norms that justify domestic and international copyright law, resulting to a guaranteed efficient enforcement of exclusive rights on one side, while maintaining open communication on the Internet. Many cases, chiefly in the European Union, exhibit the intricacies of enforcing the communication right to people, same as the public performance right by transmission in U.S. copyright parlance, in contract of those who render hyperlinks that efficiently transfer infringing information to Internet users ${ }^{7}$.

Since hyperlinks allow access to users by providing content availability on the Internet, one may provide a hyperlink concept as a form of doing works accessible to the people. The 1996 WIPO Copyright treaties presented the making accessible right resulting to modern exclusive communication right to the people under Berne Convention. The said convention comprises communication rights to people by primary and secondary transmission via wired and wireless ways, but does not fortify these rights into a sole comprehensive and coherent article. More precisely, the 1971 Paris text shares the communication for public right across varied dispositions, abandoning many gaps both as to issuing matter covered by the right, and as to the granted exclusive right. Despite the problems, it is explicit that the communication right to people reaches acts of both initial and working recommunication. In an instance, article 11 bis, regards third parties' free-to-air and wired retransmission of protected broadcasting works. It can be vaguely argued that the communication right of Berne Convention to public reached to individualized "pull" technologies, concurrently distributed to the public "push" technologies ${ }^{7}$.

The Article 8 of the WIPO Copyright Treaty ("WCT") completes the Berne Convention blanks in regard of both to issuing matter and to the communication coverage to public right. All initial and subsequent working transmissions of authorship to people come within the exclusive right coverage. Article 8 gives further explanation to the public communication right via specification of comprised right of making accessible to public works in such a means that public members may acquire availability of those works 
from a location personally selected at a time. This right reached individualized on-demand ("pull") communications (by any technical ways), for it makes an explicit that public members may be severed both in space and time. The WCT does not describe the public as member composition but implies that it must not denote a populous notion, even though the work is made accessible in large numbers, it must have an apparent conclusion that its availability was made to public ${ }^{7}$.

\section{CONCLUSIONS AND RECOMMENDATION}

The Internet is the most effective communication resources for e-learning, e-commerce and entertainment. For enhancing and promoting research proficiency, Web information is the appropriate tool for article citations over Web design. Internet sites provide several data in which the quality and credibility tend to vary by location, between subscription-based databases of highimpact journals from prestigious research institutions to mainstream articles of developing countries, as well as its small journals. Old URLs tend to be unavailable as time function as its identifier is not permanent, unlike DOls and PURLs, which are uniquely autonomous on the location of an electronic document. As a result, retrieval from Internet Archives and Google services may lead to copyright infringement of intellectual property as majority of its old URLS would fail to render complete public communication right as enforced by hyperlinks under Article 8 of WIPO Copyright Treaty. Hence, modern DOls and PURLs are recommended for article citations as their identifiers are unique and permanent, unlike old URLs that decay over time leading to inaccessibility of complete data for most of its e-learning materials.

\section{REFERENCES}

1. Saberi, MK, Abedi, H, (2012). Accessibility and decay of web citations in five open access ISI journals, Internet Research, Vol. 22 (2), pp. 234-247.

2. Matheus, A, (2004). Web Design Quality Versus Web Information Quality, Proceeding of the Ninth International Conference on Information Quality (ICIQ-04), pp. 89-98.
3. Sahin, YG, Balta, S, Ercan, T, (2010). The Use of Internet Resources by University Students During Their Course Projects Elicitation: A Case Study, The Turkish Online Journal of Educational Technology, Vol. 9 (2), pp. 234-244.

4. Habibzadeh, P, (2013). Decay of References to Web sites in Articles Published in General Medical Journals: Mainstream vs Small Journals, Applied Chemical Informatics, Vol. 4, pp. 455-464.

5. Ducut, E, Liu, F, Fontelo, P, (2008). An Update on Uniform Resource Locator (URL) decay in MEDLINE abstracts and measures for its mitigation, BMC Medical Informatics and Decision Making, 8:23, pp. 1-8.

6. Wren, JD, Johnson, KR, Crockett, DM, Heilig, LF, Schilling, LM, Dellavalle, RP, (2006). Uniform Resource Locator Decay in Dermatology Journals, Arch Dermatol, Vol. 142, pp. 1147-1152.

7. Ginsburg, JC, Budiardjo, LA, (2018). Liability for Providing Hyperlinks to Copyright-Infringing Content: International and Comparative Law Perspectives, Liability and Hyperlinks to Infringing Content, Vol. 41, pp. 153-225. 